\title{
Theoretical Investigation of Influence of Pore Pressure on Mechanical Response of Gas-Filled Permeable Materials
}

\author{
Sergey Astafurov ${ }^{1,2, a)}$, Evgeny Shilko ${ }^{1,2, b)}$, Andrey Dimaki ${ }^{1,2, c)}$, and \\ Sergey Psakhie ${ }^{1,3, d)}$ \\ ${ }^{1}$ Institute of Strength Physics and Materials Science SB RAS, Tomsk, 634055, Russia \\ ${ }^{2}$ National Research Tomsk State University, Tomsk, 634050, Russia \\ ${ }^{3}$ National Research Tomsk Polytechnic University, Tomsk, 634050, Russia \\ a) Corresponding author: svastafurov@gmail.com \\ b) shilko@ispms.tsc.ru \\ c) dav@ispms.tsc.ru \\ ${ }^{\mathrm{d})} \mathrm{sp} @$ ispms.tsc.ru
}

\begin{abstract}
The paper is devoted to theoretical investigation of the influence of gas pore pressure on the characteristics of mechanical response of gas-filled permeable materials and media. Investigation was based on computer-aided simulation by hybrid cellular automaton method. Mechanical response of the model gas-filled samples of young brown coal under unconfined (in absence of constraint) and constrained conditions was investigated. The simulation results showed that increase of the pore pressure of the gas acting on the solid skeleton leads to decrease in materials strength. This is due to the fact that the gas pressure makes an additional contribution to integral pressure acting in volume of the loaded medium. Consequences of this are earlier beginning of plastic deformation and fracture of the material. It should be noted that in the constrained conditions decreasing of material strength with increasing gas pore pressure has more pronounced nonlinear character, in comparison with similar tests for unconfined samples. This is due to the fact that loading of constrained material is accompanied by its massive cracking and, consequently, by a grater decrease of the strength characteristics of the medium.
\end{abstract}

Keywords: gas, gas-filled permeable materials, pore pressure, computer-aided simulation, hybrid cellular automaton method, fracture, strength

\section{INTRODUCTION}

Investigation of regularities of mechanical response of gas-filled permeable materials (including geological ones) is an actual problem of mechanics of solids. For example, the presence of the gas phase in the fractured pore space of geological medium (for example, in the coal-bed) could greatly influence on the strength and deformation characteristics of the material $[1,2]$. Influence of the gas phase on the parameters of mechanical response of the medium is largely determined by the peculiarities of the stress state of the system (in particular by presence or absence of constrained conditions). So the paper is devoted to a theoretical investigation of the influence of pore pressure on the mechanical response of gas-filled permeable materials in unconfined (in absence of constraint) and constrained conditions. It should be noted that investigation of influence of gas phase on the mechanical response of solid skeleton is a fairy complicated problem due to the complex mechanical interaction of phases that are in different aggregation states. Therefore, solution of this problem requires to use advanced methods of computer-aided simulation. Investigations were based on computer-aided simulation by hybrid cellular automaton method [3]. This method combines the formalisms of movable cellular automaton method $[3,4]$ (to describe the mechanical response of solid skeleton) and classical cellular automaton method (to simulate the processes of filtration and diffusion of gas in fractured pore space of the medium. This approach allows to describe deformation and fracture of gas-filled

(C) 2014 AIP Publishing LLC 978-0-7354-1260-6/\$30.00 
porous elastic-plastic medium with regard to its structure, the gas mass transfer, as well as the influence of gas pressure on the mechanical response of the solid skeleton.

\section{RESULTS OF COMPUTER-AIDED SIMULATION}

Deformation and fracture of lignite (young brown coal) [5] filled with carbon dioxide was simulated in the paper. Influence of pore pressure on the mechanical response of material in unconfined and constrained conditions. In the first case (unconfined conditions) uniaxial compression test of lignite samples was simulated. Here gas pressure in the sample ambient space was equal to $0 \mathrm{MPa}$. In the second case uniaxial compression test of constrained lignite samples was considered (loading scheme is shown in Fig. 1(a)). Model lignite sample (1) was placed between gastight lateral walls (2). The bottom surface of the sample (3) was fixed $\left(V_{y}=0\right)$. Loading of the sample was conducted by movement of upper loading element (4) with a constant low speed $V_{\text {load. }}$. Constraint of the sample was specified by application of constant force to the lateral surfaces of gas-tight walls. Specific value of this force $\sigma_{\text {lit }}$ corresponded to lithostatic pressure acting in the coal-bed at a depth of $100 \mathrm{~m}$.

In modeling of mechanical response of the gas-filled permeable medium in the framework of hybrid cellular automaton method each fragment of simulated system was represented as an ensemble of interacting movable cellular automata. An incremental theory of plasticity of isotropic medium with Drucker-Prager-Nikolaevsky plasticity criterion was used $[3,4,6]$ to model deformation of solid skeleton. Solution of elastoplastic task was carried out in two-dimensional formulation (in the approximation plane strain state). Elastoplastic response function with a two-step linear hardening was used for lignite (curve 1 in Fig. 1(b)). When modeling of constrained samples a linear response function (curve 2 in Fig. 1(b)) was used for lateral walls. This response function corresponds to a high-strength elastically deformed material. Elastic and physical parameters of the material of the lateral walls (Young's modulus, Poisson's ratio, density) were equal to corresponding characteristics of lignite. A modified Drucker-Prager fracture criterion, taking into account the influence of the local gas pore pressure on the medium strength was used:

$$
\sigma_{\mathrm{DP}}=0.5(a+1) \sigma_{\text {int }}+1.5(a-1)\left(\sigma_{\text {mean }}+P_{\text {gas }}\right)>\sigma_{\text {crit }} .
$$

Here $\sigma_{\text {crit }}$ is critical value of strength of interautomata bond, $a=\sigma_{\mathrm{c}} / \sigma_{\mathrm{t}}$ is a ratio of compressive strength $\left(\sigma_{\mathrm{c}}\right)$ to tensile strength $\left(\sigma_{\mathrm{t}}\right)$ of material, $\sigma_{\text {int }}$ and $\sigma_{\text {mean }}$ are local values of equivalent stress and mean stress respectively, $P_{\text {gas }}$ is the gas pore pressure.

The simulation results showed that under uniaxial compression unconfined samples demonstrate brittle response that in good agreement with the behavior of real lignite samples of cylindrical shape. Destruction occurs through the formation of one or several diagonal cracks. Calculations performed for different values of gas pore pressure in the range from 0 to $5.5 \mathrm{MPa}$, showed that the strength of the samples decreased with increasing of value of $P_{\text {gas }}$ by a linear law (Fig. 2). The revealed decrease in strength of gas-filled material with increasing of gas pore pressure is connected with the fact that this parameter $\left(P_{\text {gas }}\right)$ has a significant influence on the mean stress acting in the medium. Consequence of this is a more rapid advance of a moment of plastic deformation of the material, as well as decrease in its strength and ultimate strain. One example is influence of $P_{\text {gas }}$ value on strength of material.

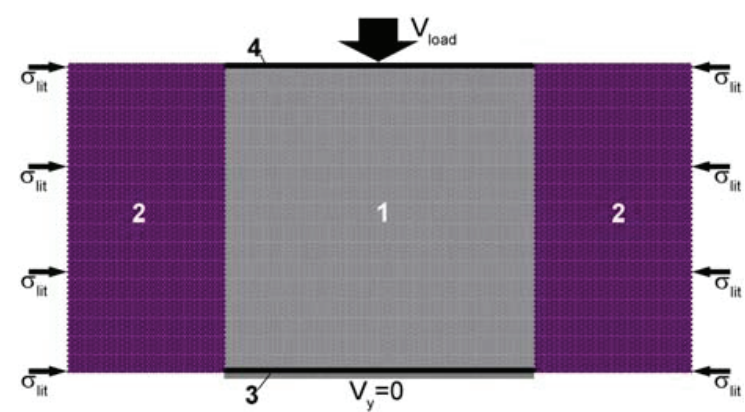

(a)

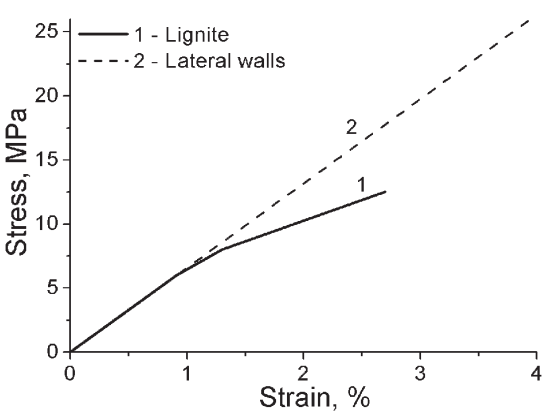

(b)

FIGURE 1. Loading scheme of the constrained sample (a) and response functions of movable cellular automaton of the components of simulated system (b). In figure (a): 1 - lignite sample; 2-gas-tight lateral walls; 3 -immobile base; 4 - loading element 


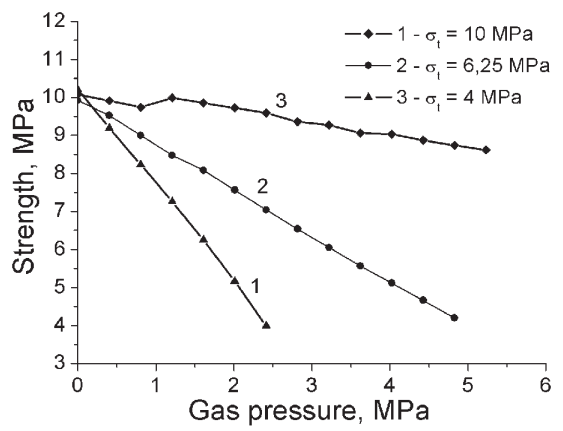

FIGURE 2. Dependences of strength of gas-filled samples on the gas pressure for different values of tensile strength of lignite

From the formulation of Drucker-Prager fracture criterion (Eq. 1) follows that an increase in pore pressure contributes additive in summand which is connected with mean stress. This leads to more rapid achievement of local strength in pair of interacted cellular automata and, hence, to decrease in integral strength of material.

It is well known that brittle materials, including most rocks, are characterized by low values of tensile strength $\left(\sigma_{t}\right)$ at sufficiently high values of compressive strength $\left(\sigma_{c}\right)$. The ratio $\sigma_{c} / \sigma_{t}=a$ in Eq. 1 can be 10 or even more. It is determined by features of heterogeneous structure of material, including the initial hierarchy of damages. Thus, with increasing of concentration of "large" defects and damages the tensile strength of the material is decreased much faster than the compressive strength. It is connected with differences in the functioning of the planar-type damages under tension and compression. Therefore, analysis of influence of the pore pressure of the gas on the strength of gas-filled permeable geological materials characterized by different ratio of $\sigma_{\mathrm{c}} / \sigma_{\mathrm{t}}=a$ is an important task. In this paper, this factor was investigated for unconfined lignite samples (Fig. 2) under uniaxial compression. Mechanical response of lignite samples with different ratios of $\sigma_{c} / \sigma_{t}=a$ was analyzed. The value of compressive strength $\left(\sigma_{\mathrm{c}}\right)$ in all calculations was considered as constant parameter $(12.5 \mathrm{MPa})$. The value of the second parameter $\left(\sigma_{t}\right)$ varied from 4 to $10 \mathrm{MPa}$. Fig. 2 shows the dependences of the strength of the simulated samples with different values of $\sigma_{t}$ on the value of the gas pore pressure $\left(P_{\mathrm{gas}}\right)$. As could be seen, these curves have a form close linear and the influence of gas pore pressure on the strength of the samples decreases with increasing of $\sigma_{\mathrm{c}} / \sigma_{\mathrm{t}}$ ratio. This is manifested, in particular, in the reducing of the slope of the curves in Fig. 2.

Fig. 3(a) shows the diagrams of uniaxial compression of constrained lignite samples, which are characterized by different values of the gas pore pressure. It is seen that the increase in pore pressure in constrained model samples leads to decrease in their strength and ultimate strain.

Analysis of simulation results showed that in contrast to unconfined samples this decrease has nonlinear character. Fig. 3(b) shows the dependence of the sample strength on the value of the gas pore pressure. It could be seen that the rate of strength decreasing of constrained samples increases significantly at $P_{\text {gas }}>1.6 \mathrm{MPa}$. This is due to the difference in the fracture character in unconfined and constrained samples.

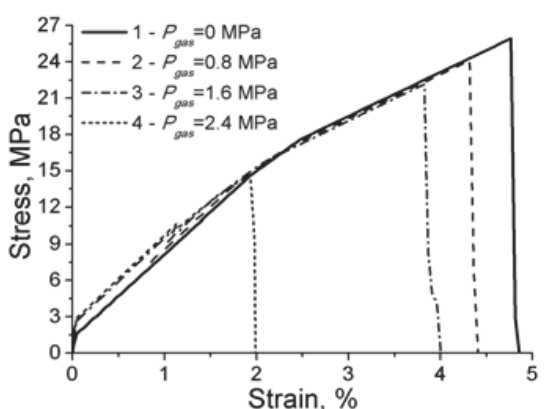

(a)

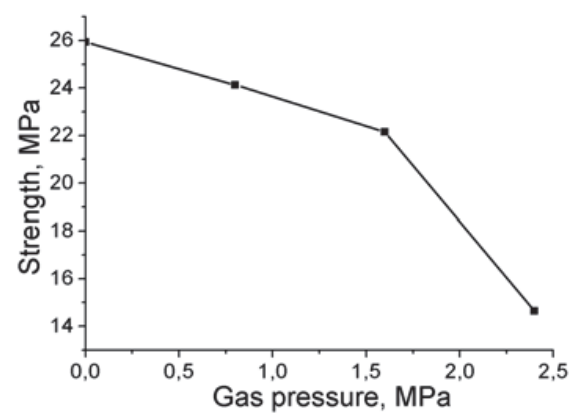

(b)

FIGURE 3. Loading diagrams of model constrained samples for different values of gas pore pressure (a) and dependences of sample strength on the gas pore pressure 
Thus along with the formation of diagonal main cracks in the volume of the material a relatively large amount of damages are formed in conditions of lateral constraint. Amount of these damages greatly increases with increasing of the pore pressure. As a result, at high values of $P_{\text {gas }}\left(P_{\text {gas }}>1.6 \mathrm{MPa}\right)$ this factor makes an additional contribution to the decreasing of the strength of gas-filled lignite samples.

It should be noted that the strength and value of the ultimate strain of constrained samples is significantly higher than the corresponding values for the unconfined material. Comparison of Fig. 2 and Fig. 3(b) shows, in particular, that even the strength of the constrained sample up to 3 times higher than for unconfined one. This fact is in good agreement with the available experimental data on the mechanical response of the deformable medium in the constrained conditions.

\section{SUMMARY}

Thus, results of computer-aided simulation showed that increase of the pore pressure of the gas acting on the solid skeleton leads to decrease in materials strength. This is due to the fact that the gas pressure makes an additional contribution to integral pressure acting in volume of the loaded medium. Consequences of this are earlier beginning of plastic deformation and fracture of the material. It should be noted that in the constrained conditions decreasing of material strength with increasing gas pore pressure has more pronounced nonlinear character, in comparison with similar tests for unconfined samples. This is due to the fact that loading of constrained material is accompanied by its massive cracking and, consequently, by a greater decrease of the strength characteristics of the medium.

The investigation has been carried out within the SB RAS Program III.23.1.4 for Basic Research and at partial financial support of the project 25.4 of Presidium of RAS.

\section{REFERENCES}

1. Y. Cao, D. He, and D. C. Glick, Int. J. Coal Geol. 48, 47 (2001).

2. J. W. Larsen, Int. J. Coal Geol. 57, 63 (2004).

3. S. G. Psakhie, E. V. Shilko, A. Yu. Smolin, A. V. Dimaki, A. I. Dmitriev, Ig. S. Konovalenko, S. V. Astafurov, and S. Zavshek, Phys. Mesomech. 14(5-6), 224 (2011).

4. S. G. Psakhie, E. V. Shilko, A. Yu. Smolin, S. V. Astafurov, and V. E. Ovcharenko, Fract. Struct. Integrity 24, 26 (2013).

5. M. Markic and R. F. Sachsenhofer, Int. J. Coal Geol. 33, 229 (1997).

6. I. A. Garagash and V. N. Nikolaevskiy, Adv. Mech. 12, 131 (1989). 